\title{
Interference effects in nonlinear Compton scattering due to pulse envelope
}

\author{
B. King๑* \\ Centre for Mathematical Sciences, University of Plymouth, Plymouth PL4 8AA, United Kingdom
}

(Received 16 December 2020; accepted 1 February 2021; published 25 February 2021)

\begin{abstract}
Nonlinear Compton scattering is calculated for the collision of an electron with a plane-wave pulse. A midinfrared (IR) peak arises in the photon spectrum due to long-range interference associated with the pulse envelope. The case of a flattop pulse is studied as a toy model for pulse envelope effects and reduced to two final-state momentum integrations; the case of a sine-squared pulse is studied numerically. A perturbative expansion in charge-field coupling reveals that already at intermediate intensities, many orders are required to correctly capture the structure of the mid-IR peak. By regularizing the classical result, it is shown that the mid-IR peak is due to plane-wave ponderomotive effects from the pulse envelope. Finally, it is shown that the mid-IR peak can be isolated using energy, angle and polarization filters.
\end{abstract}

DOI: 10.1103/PhysRevD.103.036018

\section{INTRODUCTION}

The amplitude for a probe particle to undergo a given process in a laser pulse is proportional to an integral over all spacetime. When the probability is obtained from this by mod squaring, one captures interference between the process occurring in different parts of the pulse. Here we will consider nonlinear Compton scattering [1,2], which is the emission of a photon by a highly relativistic electron in a classical background of arbitrary intensity. We will assume the interaction with a laser pulse is well approximated by a plane-wave background of finite duration, as is by now standard [3-7]. Then only the integral over the laser pulse's light front direction is nontrivial, and interference can be parametrized using the laser pulse phase.

Various approximations have been devised to define a "probability rate," which, when integrated over the entire pulse, gives the total probability for the process to occur. Such a rate is useful, as it can be added to Monte Carlo generators in particle-in-cell simulations of bunches of particles colliding with more complicated (e.g., focused) laser pulses, potentially describing each particle undergoing multiple processes [8-18]. The form of the probability rate approximation can be classified by the length scale of interference that it includes.

In a constant plane-wave field the interference between emission at different points in the background cancels exactly, such that the total probability can be written as the

\footnotetext{
*b.king@plymouth.ac.uk
}

Published by the American Physical Society under the terms of the Creative Commons Attribution 4.0 International license. Further distribution of this work must maintain attribution to the author(s) and the published article's title, journal citation, and DOI. Funded by SCOAP. integration over the laser pulse phase, of a completely local, instantaneous probability rate describing pointlike emission [19]. Therefore the emission in "slowly varying" laser pulses is often calculated using a locally constant field approximation (LCFA), which takes the completely instantaneous constant field rate and integrates it over the form of the nonconstant laser pulse field [1,19]. (A more precise definition of slowly varyin' has been recently investigated in several works [20-24].)

A more accurate but less versatile approximation of the spectrum can be acquired by perturbing around a monochromatic field. The periodic nature of a monochromatic field includes interference between emission in different cycles of equal amplitude. By including this interference exactly and approximating interference due to neighboring cycles having different amplitudes because of the pulse envelope, one can obtain a locally monochromatic approximation (LMA) [25-27], which evaluates a monochromatic "rate" at the local amplitude of the pulse envelope and integrates it over the entire pulse envelope. Such an approach reproduces harmonic structure in the photon spectrum, which is beyond the LCFA, since the harmonic structure is due to the interference between emission in different cycles. However, the LMA misses the full structure due to the pulse envelope.

The LCFA and LMA include, respectively, interference effects on subwavelength and wavelength scales. Here, we shall study interference effects in the nonlinear Compton process on the scale of the entire pulse length. The reason that interference can occur on spacetime scales longer than the pulse length is that the electron current does not vanish when the field vanishes. To calculate the radiation generated by the electron when it collides with a finite laser pulse, one must regularize the probability and remove the zero-field contribution. This regularization must be done in the 
classical case [28] as well as the quantum case. The regularization, which includes interference over arbitrarily large scales, leads to a broad mid-IR peak, which is nonperturbative in the charge-field coupling just like the wellknown harmonic structure. While this mid-IR peak can be seen in the numerical results of several authors [22,23,29], to the best of our knowledge, it has yet to be analyzed or discussed in detail. This is the focus of the current paper.

Interference effects in the nonlinear Compton process at the level of the pulse length have been studied in deltafunction pulses [30], in the shape of ultrashort pulses [31] and in double pulses [32]. The IR part of the photon spectrum includes radiative loop corrections [33,34] and can receive significant contributions from much higher orders of expansion in dressed vertices [35-40], which has contributed to discussion of the Ritus-Narozhny conjecture [41-45]. The motivation for wanting to better understanding this part of the photon spectrum in nonlinear Compton scattering is in part due to upcoming high-energy particlelaser experiments such as E320 [46] at SLAC and LUXE [47] at DESY which, by virtue of using low-emittance traditionally accelerated electron beams, will measure the nonlinear Compton process at a higher precision than has so far been possible. Furthermore, QED will be tested in the intermediate intensity regime of $\xi \sim O(1)(\xi$, also known as " $a_{0}$ " is the classical nonlinearity parameter equal to the work done by the field over a Compton wavelength in units of the background photon energy [19]), which is neither perturbative nor asymptotic in $\xi$. We stress that the mid-IR peak studied in this paper is beyond the LCFA and LMA methods being used to model these experiments.

Interference effects also occur in pair production, and the effect of interference on the duration of the pulse envelope has been studied in several works [48-53].

The paper is organized as follows. Section II introduces notation, gives the expressions that will be numerically evaluated, recaps regularization of $1 \rightarrow 2$ quantum electrodynamical (QED) processes in plane-wave backgrounds and defines the setup. In Sec. III, the toy model of a flattop pulse is presented, which gives an intuitive explanation of much of what will follow for the more realistic sine-squared pulse case. In Sec. IV, the light front momentum spectrum for nonlinear Compton scattering in a sine-squared pulse is analyzed, with particular emphasis on the emergence of the mid-IR peak as $\xi$ is increased above $\xi=1$ and on the failure of a truncated perturbation expansion to reproduce the entire spectrum. In Sec. V, the problem is analyzed using nonlinear classical electrodynamics and the classical motion of the electron identified that corresponds to the mid-IR peak. In Sec. VI, the angular spectrum and polarization content of the mid-IR peak is analyzed with a view to its separation from the rest of the spectrum, and in Sec. VII the paper is concluded. The Appendix contains some brief information on the classical calculation. Throughout, we will use a system of units in which $\hbar=c=1$.

\section{METHOD}

Four-momentum conservation in nonlinear Compton scattering can be written as

$$
p+\bar{n} \varkappa=q+k,
$$

where $p(q)$ is the incoming (outgoing) electron momentum, $k$ is the emitted photon momentum, $\varkappa$ is the laser pulse wave vector and $\bar{n}$ is a continuous number which indicates the momentum contributed to the process by the laser pulse background. Specifically, $\bar{n}=k \cdot p / \varkappa \cdot p(1-s)$, where $s=$ $\varkappa . k / \varkappa . p$ is the light front fraction of the emitted photon. $s$ is an important variable that will be used in the analysis of the results to parametrize the emission spectrum. For a fixed emission angle, varying $s$ is equivalent to varying energy, whereas for fixed energy, varying $s$ is equivalent to varying the emission angle.

The scattering matrix is $\mathrm{S}_{\mathrm{fi}}=(e / 2) \int d^{2} x^{\perp} d x^{+} \mathrm{S}_{r^{\prime}, r ; l}$, where $e<0$ is the electron charge and $\mathrm{S}_{r^{\prime}, r ; l}=$ $\int d \varphi \bar{\Psi}_{r^{\prime}, q} \mathbb{A}_{l, k} \Psi_{r, p}$, where $\varphi=\varkappa . x$ is the plane-wave phase, $\Psi_{r, p}$ is the Volkov wave function for an electron with spin index $r$, and $A_{l, k}$ is the emitted photon wave function in polarization state $l$. Before giving an explicit expression, we note that our calculation will use photon polarization states that are perpendicular to the laser wave vector (i.e., $\left.A_{l, k} \cdot \varkappa=0\right)$ and will mostly use the Boca-Florescu regularization [3]. Then we can write

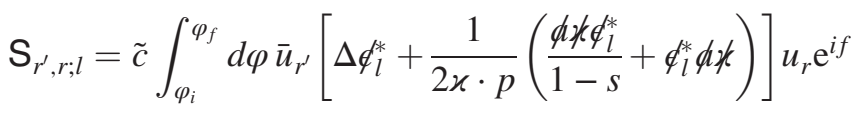

$$
f=\frac{1}{\eta(1-s)} \int_{\varphi_{i}}^{\varphi} \frac{k \cdot \Pi(z)}{m^{2}} d z
$$

where $u_{r}\left(\bar{u}_{r^{\prime}}\right)$ are the incoming (outgoing) electron bispinors, where $\eta=x \cdot p / m^{2}$ is the (electron) energy parameter, $m$ is the electron mass, $\epsilon$ is the photon polarization, $\Delta=1-k . \Pi / k \cdot p$ is the regularization factor, $\Pi$ is the classical electron kinetic momentum:

$$
\Pi=p-a+\varkappa\left[\frac{p \cdot a}{\varkappa \cdot p}-\frac{a \cdot a}{2 \varkappa \cdot p}\right]
$$

(we use the shorthand $a=e A$ with $A$ as the classical planewave pulse four-potential), and $\tilde{c}=\left[2^{3} V^{3} q^{0} k^{0} p^{0}\right]^{-1 / 2}$ is the field normalization factor. We use this form of regularization, because we will be considering a so-called "sandwich" plane-wave pulse which is defined between two definite phases (light front coordinates) $\varphi_{i} \leq \varphi \leq \varphi_{f}$ and is zero otherwise [54,55]. Hence the integration in Eq. (3) is over a finite phase interval. 
Since the regularization terms will play a crucial role, we briefly recap how they are derived (see e.g., $[3,56]$ ) and thereby show how they encode long-range interference on the order of the pulse duration. The scattering amplitude is an integral over all of spacetime, so the nontrivial integration direction along the laser pulse phase is infinite. Let us write the exponent in Eq. (3) as $f=f_{0}+f_{a}$, where $\lim _{a \rightarrow 0} f=f_{0}$, and consider only the pure phase term in the amplitude (the other parts do not require regularization). It is useful to split this integral into three pieces around where the pulse has support, which we define to be for $\varphi_{i}<\varphi<\varphi_{f}$ :

$$
\int_{-\infty}^{\varphi_{i}} \mathrm{e}^{i f_{0}^{\prime} x} d x+\int_{\varphi_{i}}^{\varphi_{f}} \mathrm{e}^{i f(x)} d x+\int_{\varphi_{f}}^{\infty} \mathrm{e}^{i f_{0}^{\prime} x+i F} d x,
$$

where

$$
F=\int_{\varphi_{i}}^{\varphi_{f}} f_{a}^{\prime}(z) d z=f_{a}\left(\varphi_{f}\right)-f_{a}\left(\varphi_{i}\right)
$$

is a phase accumulated by the electron from passing through the laser pulse (see e.g., [32] for how this accumulated phase can impact nonlinear Compton scattering). When evaluated, the two integrals where the pulse has zero support $\left(\varphi<\varphi_{i}\right.$ and $\left.\varphi>\varphi_{f}\right)$ are assumed to converge as the lower (upper) bound tends to negative (positive) infinity (e.g., through use of the standard "ie" prescription [57]). Then using integration by parts, the three integrals can be written:

$$
-\int_{\varphi_{i}}^{\varphi_{f}} \mathrm{e}^{i f(x)} \frac{d}{d x} \frac{1}{i f^{\prime}} d x
$$

Undoing the integration by parts step and rewriting factors then gives the final result

$$
\int_{\varphi_{i}}^{\varphi_{f}} \Delta \mathrm{e}^{i f(x)} d x ; \quad \Delta=1-\frac{f^{\prime}}{f_{0}^{\prime}} .
$$

[If the pulse contains no dc components, the integration by parts can be skipped and Eq. (4) can be acquired directly by choosing the lower bound of the integral in $f(x)$ to be equal to $\phi_{i}$.] Therefore, the $\Delta$ regularization terms encode the contribution over length scales that are of the order of the pulse duration and beyond.

Using Eq. (2), the total unpolarized probability can then be written as $\mathrm{P}=\alpha \mathcal{I} / \eta$, where $\alpha \approx 1 / 137$ is the finestructure constant and

$$
\begin{aligned}
\mathcal{I} & =\frac{1}{2} \sum_{r^{\prime}=1}^{2} \sum_{r=1}^{2} \sum_{l=1}^{2} \mathcal{I}_{r^{\prime}, r ; l}, \\
\mathcal{I}_{r^{\prime}, r ; l} & =\frac{1}{2^{4} \pi^{2}} \int_{0}^{1} \frac{d s}{s(1-s)} \int \frac{d^{2} k^{\perp}}{m^{2}}\left|\mathrm{~S}_{r^{\prime}, r ;}\right|^{2} .
\end{aligned}
$$

Later, we will have reason to evaluate specific polarization channels, so we will not always perform all the polarization sums in Eq. (5).

The example plane-wave pulse background that we will consider is that of a circularly polarized sine-squared pulse, which is only defined on a section of the phase line:

$$
a= \begin{cases}m \xi \sin ^{2}\left(\frac{\varphi}{2 N}\right)\left[\varepsilon_{1} \cos \varphi+\varepsilon_{2} \sin \varphi\right] & \text { if } \varphi \in[0,2 \pi N] \\ 0 & \text { otherwise }\end{cases}
$$

$N$ denotes the number of cycles and we choose lab coordinates to coincide with $\varepsilon_{1}=(0,1,0,0), \quad \varepsilon_{2}=$ $(0,0,1,0)$ and $\varkappa=\varkappa^{0}(1,0,0,1)$. Although the amplitude of the pulse and hence its intensity is actually coordinate dependent, we will refer to $\xi$ as the intensity parameter in analogy with the monochromatic result. (The monochromatic limit can be reached by letting $N \rightarrow \infty$ and extending the pulse to be defined over the entire real line, leading to $-a . a \rightarrow m^{2} \xi^{2}$.) Our results for the total probability will be characterized by the parameters: $\eta, \xi$, and $N$.

Before studying the effect of the smooth pulse envelope Eq. (6), we consider a simpler example, for which more analytical progress can be made.

\section{TOY MODEL: FLATTOP PULSE}

Let us define the "flattop" pulse by the vector potential:

$$
a= \begin{cases}m \xi\left[\varepsilon_{1} \cos \varphi+\varepsilon_{2} \sin \varphi\right] & \text { if } \varphi \in[0, \Phi] \\ 0 & \text { otherwise }\end{cases}
$$

Performing the sum in Eq. (5) to obtain the probability for unpolarized particles, the integral can be written [25]:

$$
\begin{aligned}
\mathcal{I}= & \frac{1}{(4 \pi)^{2} \eta} \int d \varphi d \varphi^{\prime} \frac{d s d^{2} \mathbf{r}^{\perp}}{m^{2} s(1-s)} \mathrm{e}^{\frac{i s}{2 \eta(1-s)} \int_{\varphi^{\prime}}^{\varphi} 1+\frac{\left(\mathbf{r}^{\perp}+s \mathrm{a}^{\perp}\right)^{2}}{m^{2} s^{2}}} \\
& \times\left[-2 m^{2} \Delta \Delta^{\prime}+g(s)\left(a^{2} \Delta^{\prime}+a^{\prime 2} \Delta-2 a \cdot a^{\prime}\right)\right],
\end{aligned}
$$

where $\quad \mathbf{r}^{\perp}=\left(\mathbf{k}^{\perp}-s \mathbf{p}^{\perp}\right) / m^{2} \quad$ and $\quad g(s)=(1-s+$ $\left.(1-s)^{-1}\right) / 2$ and, in Eq. (7) only, we use the shorthand $a=a(\varphi)$ and $a^{\prime}=a\left(\varphi^{\prime}\right)$ and similarly $\Delta=\Delta(\varphi)$ and $\Delta^{\prime}=\Delta\left(\varphi^{\prime}\right)$. By applying the Jacobi-Anger expansion [58] and following a derivation similar to the infinite monochromatic plane-wave case $[1,2,59]$, both phase integrals and the azimuthal photon momentum integral can be performed analytically, and one arrives at $\mathcal{I}=\sum_{n=n_{*}}^{\infty} \mathcal{I}_{n}$, where 


$$
\begin{aligned}
\mathcal{I}_{n}= & -\frac{\Phi}{2 \eta} \int \frac{d s d\left(r^{2}\right)}{s(1-s)} \delta_{\Phi}\left[\frac{r^{2}-r_{\infty}^{2}}{2 \eta s(1-s)}\right] \\
& \times\left\{w^{2} J_{n}^{2}(z)+\frac{\xi^{2} g}{2}\left[2 w J_{n}^{2}(z)-J_{n+1}^{2}(z)-J_{n-1}^{2}(z)\right]\right\},
\end{aligned}
$$

with $r=\left|\mathbf{r}^{\perp}\right|$, where $z=\xi r / \eta(1-s)$ and we have defined the nascent delta function

$$
\delta_{\Phi}(x)=\frac{\Phi}{2 \pi} \operatorname{sinc}^{2}\left(\frac{\Phi x}{2}\right)
$$

$[\operatorname{sinc} x=(\sin x) / x]$ and the finite-duration factor $w$ is

$$
w=\frac{s^{2}+r_{\infty}^{2}}{s^{2}+r^{2}} ; \quad r_{\infty}^{2}=2 n s \eta(1-s)-s^{2}\left(1+\xi^{2}\right) .
$$

For a finite pulse, the threshold harmonic $n_{*}$ is not constrained, i.e., $n_{*}=-\infty$. Compare this to the monochromatic, infinitely extended result familiar from the literature [1], which can be acquired by taking the limit $\Phi \rightarrow \infty$ of Eq. (8). In this limit, the background can only contribute momenta in positive integer multiples of the wave vector. For the infinite monochromatic case, the threshold harmonic is

$$
n_{*}=\left\lceil\tilde{n}_{*}\right\rceil ; \quad \tilde{n}_{*}=\frac{s\left(1+\xi^{2}\right)}{2 \eta(1-s)},
$$

where $\lceil\cdot\rceil$ is the ceiling function. In particular, we note $n_{*} \geq 1$ for the infinite monochromatic case. For the flattop pulse, the sinc function represents the spectrum of the finite-duration pulse, which, unlike the infinite monochromatic case, has a finite bandwidth. Instead of the magnitude of the photon's perpendicular momentum, $r^{2}$, being constrained to be a single value, $r_{\infty}^{2}$, it can take a range of values around this, approximately in $r_{\infty}^{2} \pm W$ with $W=\pi[\Phi / 2 \eta s(1-s)]^{-1}$. (As the sinc function has a zero at $\pi$, the bandwidth is taken to be the half-width, at approximately $\pi / 2$.) This has a consequence for which harmonics contribute to a given part of the spectrum. This can be seen by writing the finite-width transverse momentum function as

$$
\delta_{\Phi}\left(n-\tilde{n}_{*}\right),
$$

from which we see the harmonic number $n$ has support in the range approximately $n \pm \pi / \Phi$. The most significant difference to the monochromatic case occurs in two parts of the spectrum: (i) when close to the end of a harmonic range; (ii) at very low values of the light front fraction parameter $s$. Close to the end of a harmonic range, because the infinite monochromatic case constrains the threshold harmonic $n_{*}$ to integer values, the spectrum changes abruptly (e.g., the structure of the first harmonic around the highest $s$ value is often referred to as the "Compton edge" [60]); in contrast, for the finite-duration flattop pulse case, there is no such constraint on $n_{*}$ and the spectrum changes smoothly through harmonic ranges. In the infinite monochromatic case, as $s \rightarrow 0, n_{*} \rightarrow 1$ because the process is kinematically forbidden for lower values of $n$, whereas in the finiteduration case, when $s$ is sufficiently small that $\tilde{n}_{*} \lesssim 2 / \Phi$, the $n=0$ harmonic becomes accessible. In fact, the $n=0$ harmonic also corresponds to a peak, but it originates from the finite bandwidth of the pulse envelope and not from the carrier phase and is in the mid-IR range of the spectrum. In the same way that the harmonic structure arises from the nonperturbative charge-field coupling, so too does the zeroth harmonic, and its nature will be investigated in the more realistic case of a smooth pulse, in the following sections of the paper.

We illustrate the photon spectrum in a flattop pulse by evaluating Eq. (8) and normalizing the spectrum to unit IR limit. The spectrum for an intensity $\xi=2.5$, light front momentum $\eta=0.1$ and various pulse durations $\Phi=2 \pi N$ (where $N$ is the number of cycles) is plotted in Figs. 1(a) and 1(b). A comparison with the locally monochromatic approximation (LMA) and the locally constant field approximation (LCFA) is made in Fig. 1(a) and in Fig. 1(b). Two effects of shortening the pulse are demonstrated: the smoothening of harmonic edges and the shifting of the mid-IR peak to lower values of momenta.

We highlight a peculiar property of the flattop solution: if the pulse is sufficiently short, the $n=-1$ harmonic must also be included; otherwise the IR limit is incorrect. In Fig. 1(c) we show the error in the spectrum when the harmonic sum is chosen to begin at $n_{*}=1$ or $n_{*}=0$. This error increases for shorter pulses. Of course, it is kinematically forbidden for an electron to emit two photons (one from the Compton process and one "into" the field in the $n_{*}=-1$ harmonic). Instead, it is the case that, due to the finite bandwidth of photons being absorbed from the flattop pulse, also the $n=-1$ harmonic includes an absorption contribution.

To conclude this section, we state the classical result for radiation from an electron in a flattop pulse. $\mathcal{I}^{\mathrm{cl}}=$ $\sum_{n=n_{*}}^{\infty} \mathcal{I}_{n}^{\mathrm{cl}}$ where

$$
\begin{aligned}
\mathcal{I}_{n}^{\mathrm{cl}}= & -\frac{\Phi}{2 \eta} \int \frac{d s d\left(r^{2}\right)}{s} \delta_{\Phi}\left[\frac{r^{2}-\left(r_{\infty}^{\mathrm{cl}}\right)^{2}}{2 \eta s}\right] \times\left\{\left(w^{\mathrm{cl}}\right)^{2} J_{n}^{2}\left(z^{\mathrm{cl}}\right)\right. \\
& \left.+\frac{\xi^{2}}{2}\left[2 w^{\mathrm{cl}} J_{n}^{2}\left(z^{\mathrm{cl}}\right)-J_{n+1}^{2}\left(z^{\mathrm{cl}}\right)-J_{n-1}^{2}\left(z^{\mathrm{cl}}\right)\right]\right\},
\end{aligned}
$$

where $z^{\mathrm{cl}}=\xi r / \eta$, where the classical width factor $w^{\mathrm{cl}}$ is

$$
w^{\mathrm{cl}}=\frac{s^{2}+\left(r_{\infty}^{\mathrm{cl}}\right)^{2}}{s^{2}+r^{2}} ; \quad\left(r_{\infty}^{\mathrm{cl}}\right)^{2}=2 n s \eta-s^{2}\left(1+\xi^{2}\right) .
$$



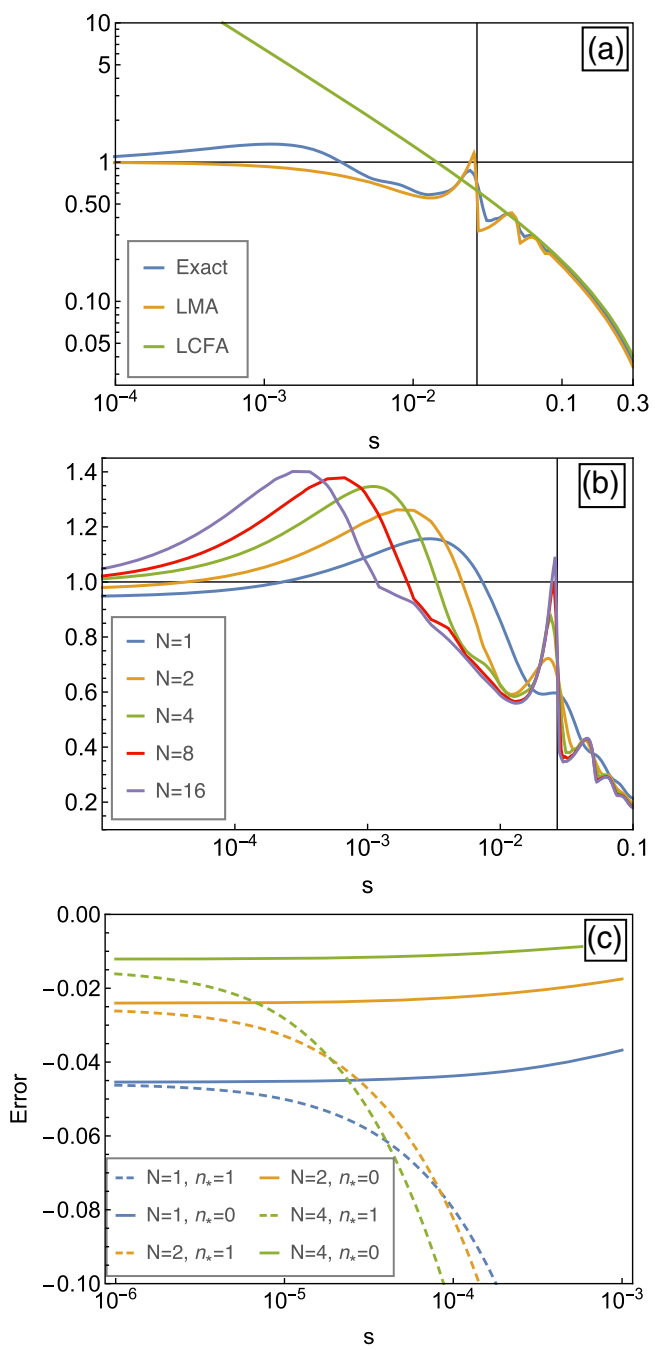

FIG. 1. (a),(b) Compton light front momentum spectra $(d \mathcal{I} / d s)$ for the flattop pulse with $n_{*}=0$. The amplitude has been normalized to unit IR limit $\left(\xi^{2} \Phi / 2\right) . N$ is the number of laser cycles $[N=4$ in (a)]. In (c), the error in the normalized spectrum is plotted, for when a different lowest harmonic, $n_{*}$, is chosen for the harmonic sum: $\mathcal{I}\left(n_{*}=-1\right) / \mathcal{I}\left(n_{*}\right)-1$.

As one might expect, Eq. (10) produces a mid-IR peak that is indistinguishable from the quantum theory but with harmonic edges at $s_{n}^{\mathrm{cl}}=2 n \eta /\left(1+\xi^{2}\right)$, which correspond to vanishing electron recoil. The agreement at small $s$ with the quantum theory motivates the classical analysis in Sec. V.

\section{LIGHT FRONT SPECTRUM}

We turn now to the more realistic sine-squared pulse envelope from Eq. (6) and illustrate the photon spectrum of nonlinear Compton scattering by evaluating Eq. (5) numerically. We calculate the quantity $\mathrm{K}=(1 / C) d \mathcal{I} / d s$ for various intensities, where the normalization factor $C$ is chosen so that $\lim \mathrm{K}_{s \rightarrow 0}=1\left(C=\frac{1}{2} \int \mathbf{a}^{2} d \varphi\right.$, which for the sine-squared pulse is equal to $\left.3 N \pi \xi^{2} / 8[23,25]\right)$. This allows for an easier comparison of spectral features. We take $\eta=0.1$, which, for example, for electrons colliding with a $1.55 \mathrm{eV}(800 \mathrm{~nm})$ laser pulse at an angle of $20^{\circ}$ to head on, corresponds to an electron energy of $8.7 \mathrm{GeV}$. The spectrum is plotted in Fig. 2 for a range of $\xi$ values: $0.1 \leq \xi \leq 4$. For small values of $\xi \sim O(0.1)$, the only significant structure is the first harmonic and the global maximum is the IR limit. As $\xi$ increases, so too does the relative amplitude of higher harmonics. As $\xi$ is increased above $\xi \approx 1$, we see a new peak develop, which, being higher than the IR limit, becomes the global maximum. This structure is what we will refer to as the "mid-IR peak." Its appearance only when $\xi \gtrsim 1$ implies that a calculation that is accurate to all orders in $\xi$ is necessary. The position of the mid-IR peak can be approximated by taking the

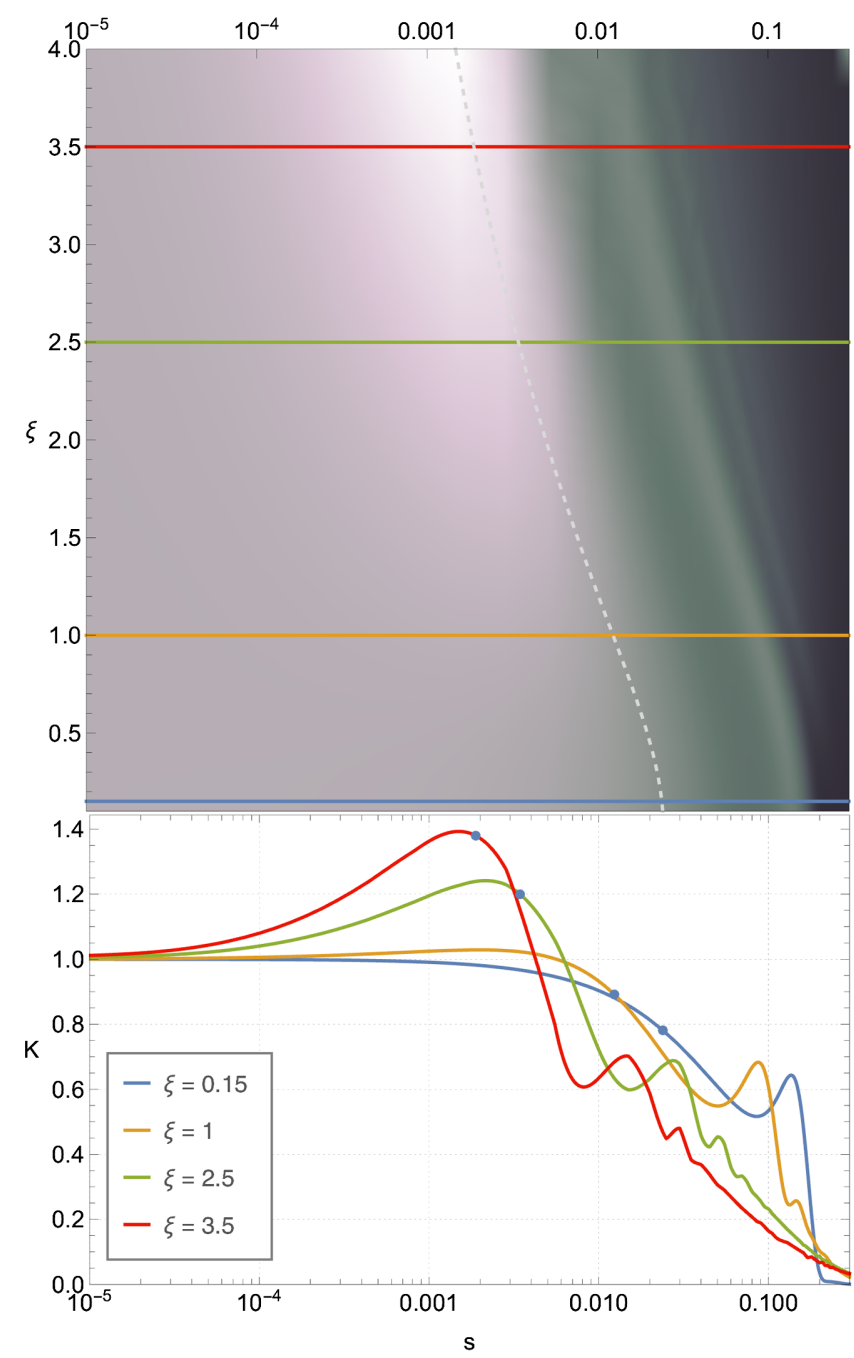

FIG. 2. How the mid-IR peak emerges as $\xi$ is increased. The horizontal axis on both figures is identical. The curves in the lower plot correspond to the lineouts indicated by solid lines on the upper plot. The dashed line is the approximate position of the mid-IR peak, which is shown on the lineouts by a dot on each curve. The results are for an $\eta=0.1$ electron colliding head on with a four-cycle $(N=4)$ pulse. 
formula for the $n$th harmonic of the monochromatic case, $s_{n}$, where

$$
s_{n}=\frac{2 n \eta}{2 n \eta+1+\xi^{2}}
$$

setting $n=1$ and rescaling $\eta \rightarrow \tilde{\eta}=\eta / 2 N$ corresponding to a wave vector with wavelength equal to the duration of the envelope. (This value of the approximate bandwidth is equivalent to that in the flattop pulse toy model studied in Sec. III.) This corresponds to replacing the carrier frequency wave vector with the wave vector of the envelope (which has a wavelength $4 \pi N$, which is $2 N$ times larger than for the carrier wave). The position of this approximation is indicated by the dashed line (dots) in the upper (lower) plot of Fig. 2. The mid-IR peak is rather broad, and this method for locating the position does not exactly coincide with the maximum. This is perhaps unsurprising: the flattop pulse case showed how a broad bandwidth of frequencies arises from the pulse envelope.

As $\xi$ increases above $\xi \approx 1$, the first harmonic grows as $d \mathrm{P} / d s \sim \xi^{2}$, but the mid-IR peak increases as $d \mathrm{P} / d s \sim \xi^{3}$. This is shown for various pulse durations in Fig. 3 (numerical results indicate the growth with $\sim \xi^{3}$ is independent of $\eta$ ). (For $N=2$, the height of the peak appears to grow slightly differently with $\xi$ than it does for longer pulses.)

To investigate the peak's nonlinear nature, we perturbatively expand $\mathrm{K}$ in the nonlinearity parameter $\xi$. Now, it is clear that, although the magnitude of the exponential is bounded, the magnitude of a series in $\xi$ of this exponential that is truncated at any finite order will be unbounded. If the expansion in $\xi$ is performed at the probability level and the ie method of regularization is used [57], this leads to difficulties in the evaluation of the zero-field limit pure phase terms. Therefore, we perform the perturbative expansion at the amplitude level given in Eq. (2), where the $j$ th perturbative order can be understood as including from the background $j$ interactions ("photons") with the electron. [At the probability level, this only corresponds to consistently including up to order $O\left(\xi^{(j+1)}\right)$ terms].

Before analyzing the mid-IR peak, we first analyze the harmonic structure for the case $\xi=0.5$ (with $\eta=0.1$ and

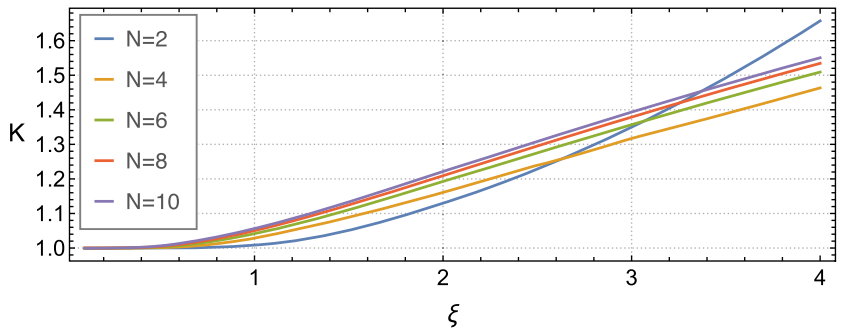

FIG. 3. The relative height of the mid-IR peak compared to the IR limit $\left(\propto \xi^{2}\right)$ grows linearly $(\propto \xi)$ when $\xi \gtrsim 1$.
$N=16$ ), where we expect the perturbative expansion of $\mathrm{K}$ to be accurate (since $\xi^{2}<1$ ). The results are presented in Fig. 4.

The first conclusion from Fig. 4 is that the harmonic order is not equivalent to how many background "photons" are interacting with the electron. In fact, even the 16th order of charge-background coupling was not sufficient to reproduce the second harmonic. Rather, the harmonic order is the "net" number of photons absorbed from the background. This is maybe unsurprising but worth emphasizing. A harmonic expansion is often acquired using the Jacobi-Anger identity to rewrite exponentials of sinusoidal functions. For example, in a circularly polarized monochromatic background, the terms linear in $a$ in Eq. (3) contribute in the factor

$$
\mathrm{e}^{-i z \sin (\varphi-\psi)}=\sum_{n=-\infty}^{\infty} J_{n}(z) \mathrm{e}^{-i n(\varphi-\psi)}
$$

where $z=\xi\left|\mathbf{r}^{\perp}\right| / \eta(1-s)$.

So we see that, expanding in $\xi$, even the first harmonic $j=1$ term is a sum over an infinite number of orders in $\xi$. Therefore, experiments that have measured second and third harmonics from nonlinear Thomson scattering (e.g., [61] where $\xi=0.5 \ldots 0.7$ and [62] which observed a $\xi^{2}$ scaling of the spectral peak position for $\xi=0.83$ ) have already measured very high nonlinear orders of interaction between the electron and the laser background [as well as [63], which measured a nonlinear scaling in $\xi$ in the $\xi>1$ regime (for $\xi$ up to $\xi=12$ ), which included up to the 500th harmonic].

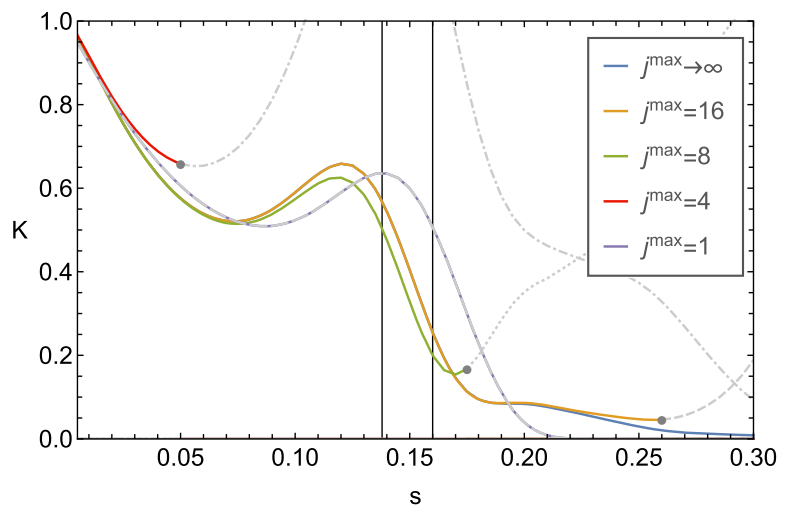

FIG. 4. Perturbative expansion of the photon spectrum for a four-cycle, $\xi=0.5$ pulse colliding with an $\eta=0.1$ electron. $j^{\max }$ corresponds to the highest order included in $\xi$ from a truncated perturbation expansion of the amplitude. (Shading and plot points have been used to guide the eye to where the perturbation expansion breaks down.) $j^{\max }=1$ corresponds to linear Compton scattering. The higher the maximum perturbative order included, the higher the value of light front momentum $s$, until which the expansion remains accurate. The vertical grid lines are the position of the nonlinear (left) and linear (right) Compton edge. 


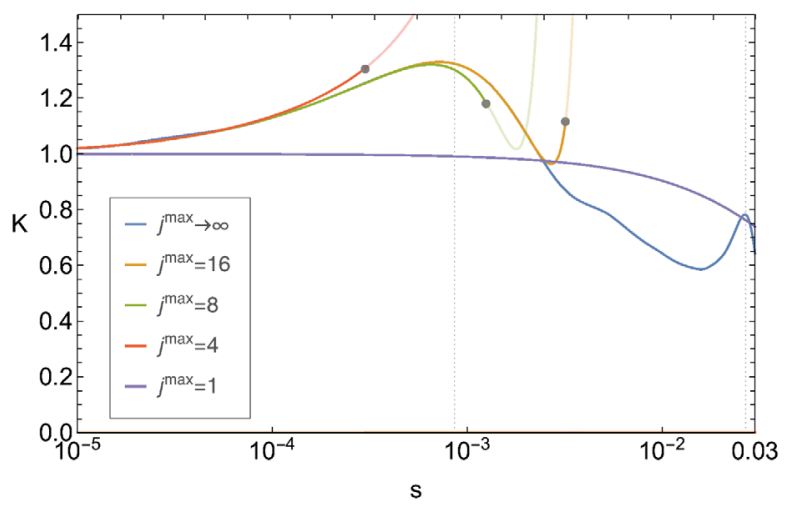

FIG. 5. Perturbative expansion of the photon spectrum for a $\xi=2.5$, 16-cycle pulse colliding with an $\eta=0.1$ electron; cf. Fig. 4. (Shading and plot points have been used to guide the eye to where the perturbation expansion breaks down.) The vertical grid lines are the estimate of the position of the mid-IR peak (left) and the first harmonic (right).

Now we turn to the mid-IR peak and increase the value of the field strength to $\xi=2.5$. On the basis of Fig. 4, one may naïvely guess that, because $s$ is very small at the midIR peak $\left[s \approx \eta / N\left(1+\xi^{2}\right) \sim O\left(10^{-3}\right)\right]$, it can compensate for the fact that $\xi^{2}>1$, allowing a low-order perturbative expansion to suffice. However, in Fig. 5 we find the opposite. Including 16 orders in the charge-field coupling is barely sufficient to reproduce the full mid-IR peak. As the mid-IR peak only appears when $\xi \gtrsim 1$, we conclude that it is formed by a highly nonlinear interaction between the electron and laser pulse background, akin to the redshifting of the Compton edge [60] and the generation of higher harmonics. This is the same way the mid-IR peak is formed in the flattop model in Sec. III, where the $n=0$ harmonic becomes accessible due to the finite pulse duration, and corresponds to a photon being absorbed with an energy of the order of the bandwidth of the laser pulse.

\section{CLASSICAL ANALYSIS}

Although highly nonlinear in the field interaction, the mid-IR peak must be a classical phenomenon. This is because the photon quantum parameter $\chi_{\gamma}=s \eta \xi$, which is a measure of the electron recoil when it emits a photon of momentum $s \eta$, obeys $\chi_{\gamma} \ll 1$. Therefore we can better understand the mid-IR peak, by asking: what part of the classical electron trajectory produces this spectral structure? The number of equivalent photons emitted classically is [57]

$$
\mathrm{N}^{\mathrm{cl}}=\int \frac{d^{3} k}{(2 \pi)^{3}} \frac{1}{2 k^{0}}|\tilde{\jmath}(k)|^{2},
$$

where the Fourier-transformed current in a plane-wave background can be written [64]

$$
\tilde{\jmath}(k)=\frac{e}{\eta m^{2}} \int d \varphi \Pi(\varphi) \mathrm{e}^{\frac{i}{\eta} \int_{-\infty}^{\varphi} \frac{k \cdot \Pi(z)}{m^{2}} d z} .
$$

Assuming $\mathrm{N}^{\mathrm{cl}} \ll 1$, the number of equivalent photons emitted classically can be interpreted as the classical limit for the probability of emission of a photon, $\mathrm{P}^{\mathrm{cl}}$. Now, the classical result will also diverge when calculated naïvely in a plane-wave pulse and must be regularized, just as in QED. Regularization of classical quantities is not a new concept (see e.g., [28]) but is relatively uncommon in the literature. We will see below how this regularization is crucial to understanding the mid-IR peak.

Regularization of the classical probability results in the phase integral being performed only over the region where the plane-wave background has support and with the momentum $\Pi$ being replaced by $\Pi^{\text {reg }}$ where

$$
\Pi^{\mathrm{reg}}=\Pi-p \frac{k \cdot \Pi}{k \cdot p} .
$$

We note that while $k \cdot \Pi \neq 0$, with the extra terms from regularization: $k \cdot \Pi^{\mathrm{reg}}=0$, which is required for current conservation $[k \cdot \tilde{j}(k)=0]$. Then the quantity that is relevant for the radiation spectrum is $\Pi^{\mathrm{reg}}(\varphi) \cdot \Pi^{\mathrm{reg}}\left(\varphi^{\prime}\right)$, which we can write as

$$
\begin{aligned}
& \underbrace{\Delta(\varphi) \Delta\left(\varphi^{\prime}\right)}_{\text {zero-field reg }}+\underbrace{a(\varphi) \cdot a\left(\varphi^{\prime}\right)}_{\perp \text { term }} \\
& \underbrace{-\left[\frac{a^{2}\left(\varphi^{\prime}\right)}{2} \Delta(\varphi)+\frac{a^{2}(\varphi)}{2} \Delta\left(\varphi^{\prime}\right)\right]}_{\| \text {term }},
\end{aligned}
$$

where the symbols $\|(\perp)$ refer to momentum directions parallel (perpendicular) to the plane-wave pulse propagation direction. In Fig. 6 we illustrate the different spectra that are produced by artificially turning off different parts of the kinetic momentum. (The method of computation for the classical calculation is briefly detailed in the Appendix.) First of all, we confirm that, as expected, the mid-IR peak is well approximated by a classical approach. Second, we find that the mid-IR peak disappears when we neglect the cross term, which is the only place the longitudinal momentum gained by the electron from the background, $\left(\xi^{2}(\varphi) / 2 \eta\right) \varkappa$, survives. The acceleration of the electron due to this term is

$$
\frac{d \Pi_{\mathrm{reg}}^{\|}}{d \tau}=\frac{m}{2} \frac{d}{d \varphi} \xi_{\mathrm{env}}^{2}(\varphi) \varkappa,
$$

where with $\xi_{\text {env }}(\varphi)$ we emphasize that for the plane-wave pulse discussed here the coordinate dependence of $\xi^{2}(\varphi)$ originates entirely from the pulse envelope $\left[\xi_{\text {env }}(\varphi) \equiv \xi(\varphi)\right.$ for a circularly polarized pulse]. Let us contrast this with the monochromatic or even locally monochromatic approach used where the "instantaneous" value of the 


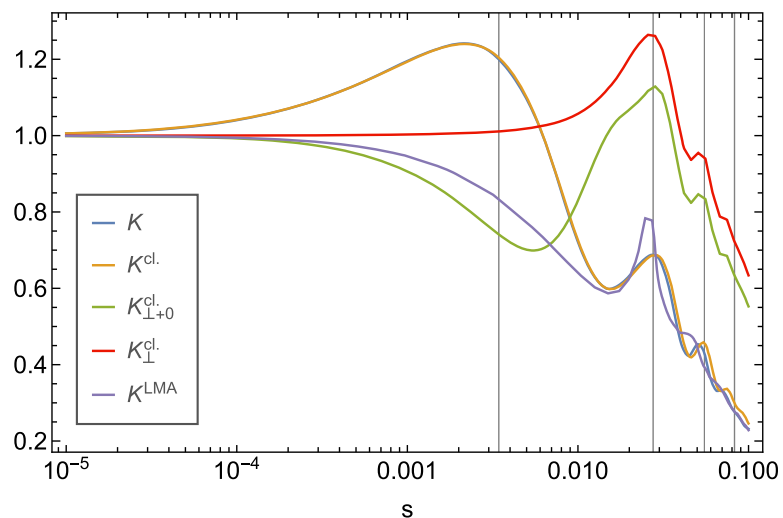

FIG. 6. Comparison of different contributions to classical radiation spectrum $(\xi=2.5, \eta=0.1, N=4)$ by removing different parts of the squared momentum in (14). $K_{\perp+0}^{\mathrm{cl}}$ corresponds to removing the longitudinal " $\|$ term" in (14), whereas $K_{\perp}^{\mathrm{cl}}$ is for where the longitudinal and zero-field terms having been removed. The LMA, which misses the mid-IR peak, is plotted for comparison. The vertical grid lines are the harmonics and the approximation to the position of the mid-IR bump.

pulse envelope is included, but higher-order derivatives are neglected [25]. In both these cases, the acceleration in Eq. (15) is zero, and there is no mid-IR peak. Therefore, we associate the mid-IR peak with this acceleration in the direction of propagation of the laser pulse, proportional to the gradient of the slow timescale of the field (i.e., the envelope), squared. The mid-IR peak can then be said to originate from a ponderomotive [65-70] effect over the scale of the pulse envelope, but in the longitudinal direction.

\section{ANGULAR SPECTRUM}

Since there is a separation in the $s$ spectrum between the mid-IR peak and the usual harmonic structure, a natural question is whether there is also a separation in the angular spectrum. Using Eq. (1), we can estimate the emission angle by taking the ratio of transverse to light front momentum of the scattered photon. We find

$$
\frac{\left|\mathbf{r}^{\perp}\right|^{2}}{s^{2} \eta^{2}}=\frac{2 \bar{n}}{\eta} \frac{1-s}{s}-\frac{1}{\eta^{2}} \geq 0 ; \quad \bar{n} \geq \frac{s}{2 \eta(1-s)}
$$

where the second inequality follows from the first. Equation (16) tells us that (i) as $s \rightarrow 0, \bar{n}$ is bounded below by 0 ; (ii) as $s \rightarrow 0,\left|\mathbf{r}^{\perp}\right| \rightarrow 0$. Therefore the mid-IR peak will feature at the center of the angular distribution.

Since $s \propto \eta_{\gamma}:=s \eta=\varkappa^{0}\left(k^{0}-k^{\|}\right) / m^{2}$, it is not immediately clear whether $k^{\|}$is positive or negative (i.e., photons scattered parallel or antiparallel to the laser propagation direction). With some rearrangement, we arrive at

$$
k^{\|}=\frac{\left|\mathbf{k}^{\perp}\right|^{2}-\kappa^{2}}{\kappa} ; \quad \kappa:=\frac{m^{2} \eta s}{\varkappa^{0}} .
$$

We see the intuitive result that, in the limit $\varkappa^{0} / m \rightarrow 0, k^{\|}$is negative; i.e., the photon is emitted antiparallel to the laser wave vector, and if $\varkappa^{0} / m \rightarrow \infty$, the photon would turn out to be emitted parallel to the laser wave vector. To acquire $\left|\mathbf{k}^{\perp}\right|^{2}$, we can calculate the angular spectrum of emitted photons. In Fig. 7, we plot an example for $s=5 \times 10^{-4}$, $\xi=2.5, N=16$ and $\eta=0.1$.

We first note that the mid-IR peak can be clearly identified in the center of the distribution. Suppose the collision between electron and laser is "head on"; then $\mathbf{r}^{\perp}=\mathbf{k}^{\perp}$. Here then $\left|\mathbf{k}^{\perp}\right| / m \sim O\left(10^{-3}\right)$, and $\eta s=5 \times 10^{-5}$. If we take an optical laser pulse, e.g., at a wavelength of $800 \mathrm{~nm}$ (energy $1.55 \mathrm{eV}$ ), then $\varkappa^{0} / m \approx 3 \times 10^{-6}$, making $\kappa / m \sim O(10)$, i.e., $\kappa \gg\left|\mathbf{k}^{\perp}\right|$, so that the photons in the mid-IR bump are scattered parallel to the electron and antiparallel to the laser direction, very close to the propagation axis. (One could consider how high a laser frequency is required for the photons to be scattered parallel to the laser direction in this case, and it would be around $25 \mathrm{keV}$.)

To filter out the mid-IR signal from the photon spectrum, ideally, one would be able to apply both an angular and an energy cut to the photons detected. The angular cut would be used to select only those photons very close to the electron propagation axis, as in Fig. 7, and the energy cut would be required; otherwise at small values of $s$, the emission from the harmonics would overlap with emission due to the mid-IR peak.

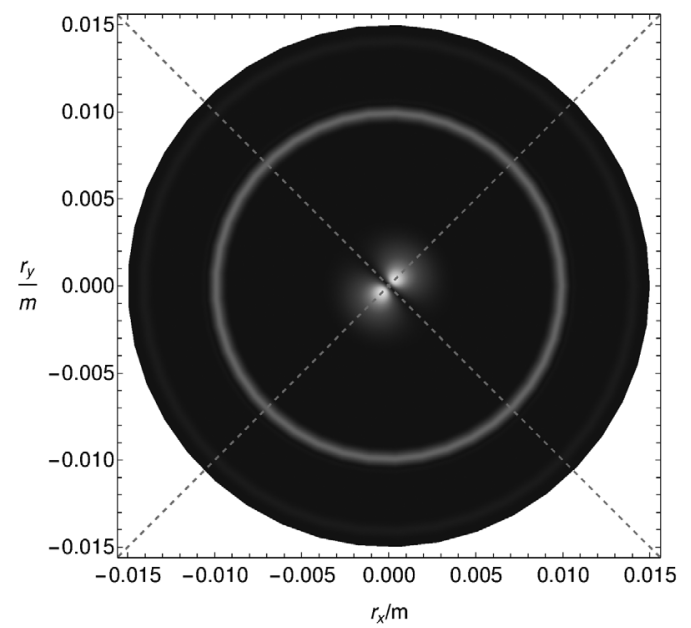

FIG. 7. A plot of the square root of the emission spectrum in the transverse, $\mathbf{r}^{\perp}$, plane, for a 16 -cycle $\xi=2.5$ pulse colliding with an $\eta=0.1$ electron producing $s=5 \times 10^{-4}$ photons (for a headon collision with $1.55 \mathrm{eV}$ laser photons, this corresponds to $4 \mathrm{MeV}$ Compton-scattered photons). The outer ring is part of the first harmonic, whereas the inner lobes are the linearly polarized signal from the mid-IR peak. Dashed lines are plotted at angles $\pm \pi / 4$ to the vertical. 


\section{(a) $\epsilon_{+}$-polarised}
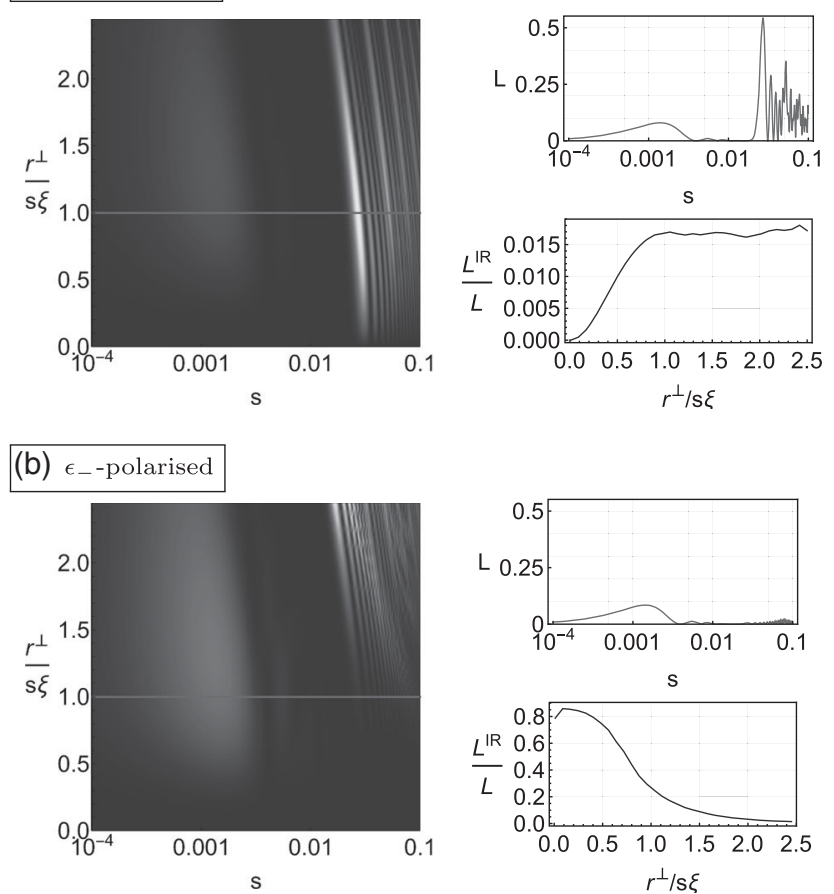

FIG. 8. Angular spectrum for the collision of a 16-cycle, $\xi=$ 2.5 pulse with an $\eta=0.1$ electron. Left: how the angular spectrum depends on photon polarization (same scale). Right upper: a lineout of the angular spectrum, $L$, at $r^{\perp}=\xi$. Right lower: proportion of $s$ spectrum of photons originating from midIR peak $L^{\mathrm{IR}} / L$ (defined as $s<0.01$ ) as a function of $r^{\perp} / \xi$.

However, another difference between the mid-IR peak photons and the rest of the spectrum is apparent from Fig. 7. In the center of the distribution, a typical dipole distribution can be seen, showing that the mid-IR peak is clearly linearly polarized, whereas the harmonics are circularly polarized. This occurs because the pulse envelope, which is responsible for the mid-IR peak, multiplies both transverse components of the background in the same way-there is no difference in the carrier-envelope phase between the two background polarization directions. Therefore the envelope imposes a linearly polarized structure on the background in contrast to the carrier frequency, which is circularly polarized. If one applies a filter to the scattered photons to separate right-handed from left-handed polarization, then the harmonics can be partially filtered out. This is demonstrated in Fig. 8, where the angular dependency is illustrated by calculating the quantity $\mathrm{L}=d \mathrm{~K} / d\left(r^{\perp} / s\right)$. We use the circular polarization basis $\epsilon_{ \pm}=\left(\epsilon_{1} \pm i \epsilon_{2}\right) / \sqrt{2}$, where

$$
\epsilon_{j}=\varepsilon_{j}-\varkappa \frac{k \cdot \varepsilon_{j}}{k \cdot \varkappa} ; \quad \varepsilon_{j}^{\mu}=\delta_{j}^{\mu}, \quad j \in\{1,2\} .
$$

To calculate the spectrum of polarized photons, we do not sum over photon polarization states, $l$, in Eq. (5), but instead just select the polarization required (recalling that $\epsilon^{*}$ enters the amplitude, and $\epsilon_{ \pm}^{*}=\epsilon_{\mp}$ ). From the results, we note that the mid-IR peak occurs with an equal amplitude in both $\epsilon_{ \pm}$circular polarization states, which is logical because linearly polarized photons are an equal mixture of circularly polarized states. But we see that, for $r^{\perp} \leq s \xi$, the signal from the harmonics is strongly suppressed in the $\epsilon_{-}$polarized photons. This can be understood writing the background pulse, Eq. (6), in terms of circular polarization vectors:

$$
a=\frac{m \xi}{\sqrt{2}} \sin ^{2}\left(\frac{\varphi}{2 N}\right)\left[\varepsilon_{+} \mathrm{e}^{-i \varphi}+\varepsilon_{-} \mathrm{e}^{i \varphi}\right] .
$$

It will be sufficient to consider the linear Compton process. In this case, a photon must be absorbed from the background; otherwise the process is kinematically forbiddentherefore only the $\varepsilon_{+}$polarization is involved from the background. In the low-s limit, the process should be well approximated by the classical formula for Thomson scattering, the probability of which is $\propto\left(\epsilon_{\text {in }} \cdot \epsilon_{\text {out }}^{*}\right)^{2}$ [64]. For photon polarization, we have chosen $\epsilon_{\text {out }}=\epsilon_{ \pm}$from Eq. (17). Suppose we consider photons being emitted mainly down the electron propagation axis, as we expect, because the electron is highly relativistic, and radiation is mainly emitted in a $\sim 1 / \gamma$ emission "cone"; then $\epsilon_{ \pm} \approx \varepsilon_{ \pm}$. Then since $\epsilon_{\text {in }}=\varepsilon_{+}$, and since $\varepsilon_{+} \cdot \varepsilon_{-}^{*}=0$, we see that emission of $\varepsilon_{-}$polarized photons in the first harmonic should be strongly suppressed, which is indeed what we find in Fig. 8. Therefore, in addition to an angular and energy cutoff, also a polarization filter could be used to isolate the mid-IR signal photon [71].

\section{CONCLUSION}

In this work, the effect of the background's pulse envelope on the spectrum of single nonlinear Compton scattering has been investigated. This corresponds to taking into account exactly interference from emissions by the electron at different points in the pulse, up to the length scale of the entire pulse envelope. This was done, first in the toy model of a flattop pulse, for which we presented the total probability as a sum over harmonics of an integral over two outgoing photon momentum components. The appearance of a spectral feature, which is absent in the wellknown infinite or locally monochromatic cases, of a harmonic peak in the midinfrared was identified. This mid-IR peak was found to originate from the finite bandwidth of the flattop pulse, which allowed the "zeroth" harmonic to become kinematically accessible. One can make an analogy, as was done for pair creation in [72], of processes in a flattop pulse being akin to emission from a diffraction grating. Here, the integer harmonics of the photon spectrum correspond to interference between the grating slits (provided by the carrier frequency of the pulse) whereas the zeroth and lower harmonics are due to 
interference from the finite width of the grating itself (provided by the flattop pulse envelope). Opening of kinematic channels due to a laser pulse's finite bandwidth is known in other strong-field QED calculations such as pair creation [72] where, in [73], using the Dirac-HeisenbergWigner formalism a ponderomotive effect on the pair spectrum was identified, real photon-photon scattering [74-77] and the linear trident process [78]. This effect, which widens harmonic fringes in the emitted photon phase space, is distinct from the opening of channels in a pulse due to just having a spacetime-dependent intensity and a variable effective mass. For this reason, the mid-IR peak is missed by local approximations, such as the LCFA and LMA.

The rest of the paper analyzed the mid-IR peak in the context of a more realistic plane-wave pulse with a sinesquared envelope. The mid-IR peak was found to be associated with a background wave vector approximately $1 / 2 N$ smaller than from the carrier frequency, where $N$ is the number of cycles of the carrier frequency. The mid-IR peak only appears when the intensity parameter $\xi$ (or " $a_{0}$ ") fulfills $\xi \gtrsim 1$, and its height in the light front spectrum grows as $\propto \xi^{3}$ compared to the IR limit, which is given by the leading-order term in $\xi$ and grows as $\propto \xi^{2}$. The mid-IR peak is a signature of an all-order interaction between the field and the charge, which was confirmed by the failure of a truncated perturbation expansion to approximate this part of the spectrum. Using a classical analysis, we were able to show that this peak arises from the ponderomotive force from the leading and trailing edges of the pulse envelope imparting a change in longitudinal momentum of the electron. Since this force is proportional to the derivative of the envelope squared, this is an alternative explanation for why the mid-IR peak is missed from local approaches such as the LCFA and LMA, which neglect derivatives of the pulse envelope. Central to this analysis was the regularization of the zero-field limit of the classical result. Therefore, an extension to this work would be to include radiation reaction [79-81]. Finally, it was shown that if the carrier frequency is circularly polarized, then the mid-IR peak, which comprises linearly polarized photons, can be partially isolated from the rest of the spectrum using polarimetry and angular cuts of low-energy photons.

\section{ACKNOWLEDGMENTS}

B. K. thanks A. Ilderton and A. Macleod for helpful discussions and comments on the manuscript. The author is supported by the UK Engineering and Physical Sciences Research Council (EPSRC), Grant No. EP/S010319/1.

\section{APPENDIX: CLASSICAL SPECTRUM CALCULATION}

Beginning with Eqs. (12) and (13), without loss of generality, we set $p \cdot \epsilon_{1,2}=0$. To obtain the spectrum, we then have

$$
\mathrm{K}^{\mathrm{cl}}=\frac{8}{3 \pi} \frac{\alpha}{N \xi^{2} \eta} \frac{1}{(2 \pi)^{2}} \int d^{2} \mathbf{r}^{\perp} \frac{T}{m^{2}},
$$

where the prefactor $8 / 3 \pi N \xi^{2}$ is chosen so that $\lim _{s \rightarrow 0} \mathrm{~K}^{\mathrm{cl}}=1$ and

$$
\begin{aligned}
T= & \underbrace{\left|\mathrm{S}_{0, \Delta}^{\mathrm{cl}}\right|^{2}}_{\text {zero-field reg }}+\underbrace{\operatorname{Re} S_{2}^{\mathrm{cl}} \mathrm{S}_{0, \Delta}^{\mathrm{cl} *}}_{\| \text {term }} \\
& \underbrace{-\left|\mathrm{S}_{1 \varepsilon}^{\mathrm{cl}}\right|^{2}-\left|\mathrm{S}_{1 \beta}^{\mathrm{cl}}\right|^{2}}_{\perp \text { term }},
\end{aligned}
$$

where

$$
\mathrm{S}_{j}^{\mathrm{cl}}=\int_{\varphi_{i}}^{\varphi_{f}} d \varphi I_{j}(\varphi) \mathrm{e}^{i(1-s) f},
$$

where $I_{0, \Delta}=\Delta, \quad I_{1 \epsilon}=-a \cdot \epsilon_{1}, \quad I_{1 \beta}=-a \cdot \epsilon_{2} \quad$ and $I_{2}=-a \cdot a$. The labeling of the terms in Eq. (A2) corresponds to the labeling in the main text in Eq. (14). The exponent function $f$ is defined in the main text in Eq. (3) and the regularization factor $\Delta=1-k \cdot \Pi / k . p$.
[1] A. I. Nikishov and V. I. Ritus, Sov. Phys. JETP 19, 529 (1964).

[2] L. S. Brown and T. W. B. Kibble, Phys. Rep. 133, A705 (1964).

[3] M. Boca and V. Florescu, Phys. Rev. A 80, 053403 (2009).

[4] D. Seipt and B. Kämpfer, Phys. Rev. A 83, 022101 (2011).

[5] F. Mackenroth and A. Di Piazza, Phys. Rev. A 83, 032106 (2011).

[6] K. Krajewska and J. Kaminski, Phys. Rev. A 85, 062102 (2012).
[7] V. Dinu and G. Torgrimsson, Phys. Rev. D 99, 096018 (2019).

[8] N. V. Elkina，A. M. Fedotov, I. Yu. Kostyukov, M. V. Legkov, N. B. Narozhny, E. N. Nerush, and H. Ruhl, Phys. Rev. ST Accel. Beams 14, 054401 (2011).

[9] C. P. Ridgers, C. S. Brady, R. Duclous, J. G. Kirk, K. Bennett, T.D. Arber, A.P.L. Robinson, and A.R. Bell, Phys. Rev. Lett. 108, 165006 (2012).

[10] B. King, N. Elkina, and H. Ruhl, Phys. Rev. A 87, 042117 (2013). 
[11] S. S. Bulanov, C. B. Schroeder, E. Esarey, and W. P. Leemans, Phys. Rev. A 87, 062110 (2013).

[12] C. Ridgers, J. G. Kirk, R. Duclous, T. Blackburn, C. Brady, K. Bennett, T. Arber, and A. Bell, J. Comput. Phys. 260, 273 (2014).

[13] A. Gonoskov, S. Bastrakov, E. Efimenko, A. Ilderton, M. Marklund, I. Meyerov, A. Muraviev, A. Sergeev, I. Surmin, and E. Wallin, Phys. Rev. E 92, 023305 (2015).

[14] T. G. Blackburn, C. P. Ridgers, J. G. Kirk, and A. R. Bell, Phys. Rev. Lett. 112, 015001 (2014).

[15] E. Gelfer, A. Mironov, A. Fedotov, V. Bashmakov, E. Nerush, I. Y. Kostyukov, and N. Narozhny, Phys. Rev. A 92, 022113 (2015).

[16] M. Vranic, T. Grismayer, R. A. Fonseca, and L. O. Silva, New J. Phys. 18, 073035 (2016).

[17] E. S. Efimenko, A. V. Bashinov, A. A. Gonoskov, S. I. Bastrakov, A. A. Muraviev, I. B. Meyerov, A. V. Kim, and A. M. Sergeev, Phys. Rev. E 99, 031201 (2019).

[18] D. Seipt, C. P. Ridgers, D. Del Sorbo, and A. G. Thomas, arXiv:2010.04078.

[19] V. I. Ritus, J. Russ. Laser Res. 6, 497 (1985).

[20] C. N. Harvey, A. Ilderton, and B. King, Phys. Rev. A 91, 013822 (2015).

[21] A. Di Piazza, M. Tamburini, S. Meuren, and C. Keitel, Phys. Rev. A 98, 012134 (2018).

[22] A. Ilderton, B. King, and D. Seipt, Phys. Rev. A 99, 042121 (2019).

[23] A. Di Piazza, M. Tamburini, S. Meuren, and C. Keitel, Phys. Rev. A 99, 022125 (2019).

[24] B. King, Phys. Rev. A 101, 042508 (2020).

[25] T. Heinzl, B. King, and A. Macleod, Phys. Rev. A 102, 063110 (2020).

[26] K. Yokoya, Report No. KEK Pub 4/96, 2003.

[27] A. Hartin, Int. J. Mod. Phys. A 33, 1830011 (2018).

[28] V. Dinu, T. Heinzl, and A. Ilderton, Phys. Rev. D 86, 085037 (2012).

[29] T. G. Blackburn, D. Seipt, S. S. Bulanov, and M. Marklund, Phys. Plasmas 25, 083108 (2018).

[30] A. Ilderton, Phys. Rev. D 100, 125018 (2019).

[31] D. Seipt, V. Kharin, S. Rykovanov, A. Surzhykov, and S. Fritzsche, J. Plasma Phys. 82, 655820203 (2016).

[32] A. Ilderton, B. King, and S. Tang, Phys. Lett. B 804, 135410 (2020).

[33] D. Yennie, S. Frautschi, and H. Suura, Ann. Phys. (N.Y.) 13, 379 (1961).

[34] S. Weinberg, Phys. Rev. 140, B516 (1965).

[35] M. Lavelle and D. McMullan, J. High Energy Phys. 03 (2006) 026.

[36] M. Lavelle and D. McMullan, Nucl. Phys. B, Proc. Suppl. 174, 51 (2007).

[37] M. Lavelle, D. McMullan, and M. Raddadi, Phys. Rev. D 87, 085024 (2013).

[38] J. P. Edwards and A. Ilderton, Phys. Rev. D 103, 016004 (2021).

[39] A. Ilderton, B. King, and S. Tang, Phys. Rev. D 102, 076013 (2020).

[40] A. Ilderton and A. J. MacLeod, J. High Energy Phys. 04 (2020) 078.

[41] A. Fedotov, J. Phys. Conf. Ser. 826, 012027 (2017).
[42] T. Podszus and A. Di Piazza, Phys. Rev. D 99, 076004 (2019).

[43] A. Ilderton, Phys. Rev. D 99, 085002 (2019).

[44] A. Mironov, S. Meuren, and A. Fedotov, Phys. Rev. D 102, 053005 (2020).

[45] T. Heinzl, A. Ilderton, and B. King, arXiv:2101.12111.

[46] S. Meuren, P. H. Bucksbaum, N. J. Fisch, F. Fiza, S. Glenzer, M. J. Hogan, K. Qu, D. A. Reis, G. White, and V. Yakimenko, arXiv:2002.10051.

[47] H. Abramowicz et al., arXiv:1909.00860.

[48] N. Abdukerim, Z.-L. Li, and B.-S. Xie, Phys. Lett. B 726, 820 (2013).

[49] M. F. Linder, C. Schneider, J. Sicking, N. Szpak, and R. Schützhold, Phys. Rev. D 92, 085009 (2015).

[50] I. A. Aleksandrov, G. Plunien, and V. M. Shabaev, Phys. Rev. D 95, 056013 (2017).

[51] F. Fillion-Gourdeau, F. Hebenstreit, D. Gagnon, and S. MacLean, Phys. Rev. D 96, 016012 (2017).

[52] G. Torgrimsson, C. Schneider, J. Oertel, and R. Schützhold, J. High Energy Phys. 06 (2017) 043.

[53] A. Ilderton, Phys. Rev. D 101, 016006 (2020).

[54] Calculations were also performed where regularization was carried out at the probability level using the $i \epsilon$ prescription, and no discernible difference was found in the resulting photon spectra.

[55] V. Dinu, Phys. Rev. A 87, 052101 (2013).

[56] A. Ilderton, B. King, and A. J. Macleod, Phys. Rev. D 100, 076002 (2019).

[57] M. E. Peskin and D. V. Schroeder, An Introduction to Quantum Field Theory (1995), ISBN 9780201503975, 0201503972, http://www.slac.stanford.edu/spires/find/ books/www?cl=QC174.45\%3AP4.

[58] G. N. Watson, Theory of Bessel Functions (Cambridge University Press, Fetter Lane, EC4, 1922).

[59] V. B. Berestetskii, E. M. Lifshitz, and L. P. Pitaevskii, Quantum Electrodynamics (Second Edition) (ButterworthHeinemann, Oxford, 1982).

[60] C. Harvey, T. Heinzl, and A. Ilderton, Phys. Rev. A 79, 063407 (2009).

[61] Y. Sakai, I. Pogorelsky, O. Williams, F. O’Shea, S. Barber, I. Gadjev, J. Duris, P. Musumeci, M. Fedurin, A. Korostyshevsky et al., Phys. Rev. Accel. Beams 18, 060702 (2015).

[62] K. Khrennikov, J. Wenz, A. Buck, J. Xu, M. Heigoldt, L. Veisz, and S. Karsch, Phys. Rev. Lett. 114, 195003 (2015).

[63] W. Yan, C. Fruhling, and G. E. A. Golovin, Nat. Photonics 11, 514 (2017).

[64] J. D. Jackson, Classical Electrodynamics (3rd Edition) (John Wiley \& Sons, Inc., New York, 1999).

[65] N. B. Narozhny and M. S. Fofanov, J. Exp. Theor. Phys. 90, 753 (2000).

[66] A. V. Gaponov and M. A. Miller, J. Exp. Theor. Phys. 7, 168 (1958).

[67] T. W. B. Kibble, Phys. Rev. 150, 1060 (1966).

[68] D. Bauer, P. Mulser, and W. H. Steeb, Phys. Rev. Lett. 75, 4622 (1995).

[69] E. A. Startsev and C. J. McKinstrie, Phys. Rev. E 55, 7527 (1997).

[70] B. Quesnel and P. Mora, Phys. Rev. E 58, 3719 (1998). 
[71] We mention for completeness sake that, because the mid-IR peak corresponds to a small recoil parameter $s \chi \ll 1$, the role of electron spin flipping is negligible, which is also evidenced by how accurately the classical theory approximated the quantum theory.

[72] T. Heinzl, A. Ilderton, and M. Marklund, Phys. Lett. B 692, 250 (2010).

[73] C. Kohlfürst and R. Alkofer, Phys. Rev. D 97, 036026 (2018).

[74] B. King and C. H. Keitel, New J. Phys. 14, 103002 (2012).

[75] H. Gies, F. Karbstein, and N. Seegert, Phys. Rev. D 93, 085034 (2016).
[76] H. Gies, F. Karbstein, C. Kohlfürst, and N. Seegert, Phys. Rev. D 97, 076002 (2018).

[77] B. King, H. Hu, and B. Shen, Phys. Rev. A 98, 023817 (2018).

[78] U. Hernandez Acosta and B. Kämpfer, Plasma Phys. Controlled Fusion 61, 084011 (2019).

[79] E. Lifshitz, The Classical Theory of Fields: Volume 2, 4th ed. (Butterworth-Heinemann, Oxford, 1987).

[80] A. Di Piazza, K. Z. Hatsagortsyan, and C. H. Keitel, Phys. Rev. Lett. 105, 220403 (2010).

[81] A. Ilderton and G. Torgrimsson, Phys. Lett. B 725, 481 (2013). 